\title{
INFORMATION STATE OF SYSTEM ESTIMATION
}

\author{
Orest Ivakhiv \\ Computer Technology, Automation and Metrology Institute, Lviv Polytechnic National University, \\ 12 Bandera str., Lviv, Ukraine, oresti@polynet.lviv.ua
}

\begin{abstract}
As far as the behavior of the object under study is random, it is difficult or even impossible to create an adequate program of its regular maintenance. A better result provides an adaptive servicing algorithm for the needs of current monitoring and control of the object. Nowadays, both different adaptive algorithms and capabilities of a sample group coding are effectively used for this purpose. The demand to the channel capacity regarding the required decrease of the binary digit rate has always been an actual task. Recently, it is observed that the creation of a new system is frequently based on the employment of the entropy measure and permutation encoding. Such applications are known to be used for biometric systems, cryptography, body wireless networks and others. A concrete combination of active source addresses (with significant samples) may be considered as some generalized image of the object under study. It can be used as information for the control function of the next level of the cyber-physical system as well. The paper deals with considering the possibility of the most efficient usage of a transmission channel capacity and receiving of a generalized image of a servicing object state as the entropy estimation of its sensors activities. The estimating operation procedures are also described. The ways of such tasks solving are described and the above mentioned coding procedure is presented. Copyright $(\mathbb{C}$ Research Institute for Intelligent Computer Systems, 2015. All rights reserved.
\end{abstract}

Keywords: entropy, information, system, coding, permutation, adaptive, activity.

\section{INTRODUCTION}

The state of the object being observed is estimated by using a set of different transducers, the so-called measurement data sources. The primary data are processed by further means of unification and the subsequent ADC. Optimal servicing program should to the utmost meet the needs of the object tracking system. Since the behavior of the object under study is random, it is difficult or even impossible to create an adequate program of regular maintenance. A better results provides an adaptive servicing algorithm for the needs of current monitoring and control of the object [1,2]. The conditional mean of the system state vector may be found by passing the conditional mean of the measurement history. There were considered stationary and non-stationary data, noiseless-channel versions of the PCM, predictive quantization, and predictive-comparison data compression systems; ensemble-average performance of the nonlinear filters was derived [3]. In particular, this can be realized using a polynomial predictor or adaptive commutator. It is capable of separating from the $\mathrm{i}$-th primary measuring signal the samples that go beyond the defined tolerance field whose value is given by the permissible quantization error. Unlike regular surveys, the flow of the samples essential for the consumer appear in a chaotic time moments, as well the order they appear from various sources is unknown. There is a need for marking the onset of the samples and identifying their affiliation (i.e., addressing) to a particular so-called active source. The concrete combination of these addresses of significant samples of active sources may be represented by some generalized image of the object under study. It can be used as information for the control function of the next level of the cyberphysical system as well [4]. If an individual address is used for source identification, then it is encoded by primitive binary code. In this case, it would require $\log _{2} n$ binary digits (here $-n$ is the number of sources beeing measured). A paradoxical situation is observed: on the one hand, the adaptive maintenance reduces the information redundancy of a system measurement data due to the availability of the real aggregate activity sources. However, cost of the service suppliment by using the individual addresses made the situation worse. An addressing of the group of non - redundant samples turns out to provide more promising results. To this end, there may be used a permutation group coding [5], because its number of symbols per one sample is close to the entropy of the active sources totality. While the use of primitive coding for individual 
source address corresponds with the same activity of each source, i.e., equal to the maximum entropy value of sources totality (i.e., equiprobable sources). Both permutation coding and entropy measure are often effectively used for wireless networks [6-9], dynamic [10, 11] and other systems [12-14], especially for biometric usage. For example, body wireless network uses an intrinsic characteristic of the human body as the authentication identity or a means of securing the distribution of a cipher key to secure inter - body area sensor network communications [6]. The relationship was studied between a novel personal entropy measure for online signatures and the performance of several state-ofthe-art classifiers which showed that there is a clear relationship between such an entropy measure both of a person's signature and the behavior of the classifier [15]. Currently, almost all systems involve an identity authentication process before a user can access the requested services such as online transactions, entrance to a secured vault, logging into a computer system, accessing laptops, secure access to buildings, etc. Therefore, authentication has become the core of any secure system, wherein most of the cases rely on identity recognition approaches. Biometric systems provide the solution to ensure that only a legitimate user and no one else access the rendered services. There was analysed the information content of the haptic data generated directly from the instrument interface [12]. It was successfully applied to the cryptography needs [16]. For some well-known chaotic dynamical systems, it is shown that the complexity of their behavior is particularly well described by entropy measure in the presence of dynamical or observational noise [7]. It was shown that the metric and permutation entropy rates - measures of new disorder per new observed value - are equal for ergodic finitealphabet information sources (i.e., discrete-time stationary stochastic processes). Finally, the equality of permutation and metric entropy rates is extended to ergodic non-discrete information sources when entropy is replaced by differential entropy in the usual way [8]. Amplitude quantization and permutation encoding are two approaches to efficient digitization of analog data [10]. The recently proposed conceptually simple and easily calculated measure of permutation entropy can be effectively used to detect qualitative and quantitative dynamical changes [17].

We propose both to use the entropy measure for the examination of the information state of object and for proper algorithm creation.

The non-redundant samples introduce some permutation set sequence [18]:

$$
p=\{x(1), x(2), \ldots, x(N)\},
$$

here, $x(j)$ is $x$-type symbol at the $j$-th position of permutation $(x=\overline{1, n} ; j=\overline{1, N})$.

It is necessary to consider two situations: the statistic of totality system sources activities is $a$ priory known and unknown $[19,20]$.

\section{STATISTICS OF SYSTEM SOURCES ACTIVITIES IS A PRIORI WELL KNOWN}

In this case, both sources set, whose shape sequence of the non-redundant samples and the amount of the granted positions are fixed. However, contrary to the regular type system, the source signal sampling is random, corresponding to the signal current behavior. At the length $N>>1$, the $i$-th non-redundant selective values will occur in the sequence boundaries $N_{i}$ times $\left(N_{i}=\alpha_{i} N\right)$. If the measurement data from $n$ sources is compressed, then the sequence versions amount is $Q_{0}=n^{N}$, because on the sequence any position can be a non redundant value of arbitrary packed multiplexed sources. Nevertheless, due to the convergence of the event frequency to its probability at a considerable amount of experiments, for $N>>1$, it is conditionally possible to arrange all the sequences into two subgroups: typical, for which $N_{i} \approx \alpha_{i} N$ and atypical - for which this relation is variable. Thus, all the typical sequences aggregate the probability with the lengths $\mathrm{N}$ increasing and will be guided to one, while the atypical sequences will tend to zero, so there will be only a necessity of the typical sequences enumeration. From the mathematical point of view, such $\mathrm{N}$-positions sequences is a permutation with the repetitions [5, $10,21,22]$, in which each $i$-th element address will occur in the sequences boundaries $N_{i}$ times. The different typical sequences number is as follows:

$$
Q=N ! / \prod_{i} N_{i} !
$$

and each corresponding number we shall present as follows:

$$
k_{\text {frame }}=\log _{2} Q=\log _{2} N !-\sum_{i} \log _{2} M_{i} !
$$

This number we shall term as a code of a disposition, the so-called Block Number Code. Having taken advantage of a Stirling formula [22] for factorials $n ! \approx r^{n} e^{-e} \sqrt{2 \pi n}$, we shall note 


$$
\ln (n !)=\frac{1}{2} \ln 2 \pi+\frac{1}{2} \ln n+n(\ln n-1), \underset{n>1}{\lim }\{\ln (n !)\} \approx n(\ln n-1)
$$

and now

$$
k_{\text {frame }}=k_{Q}=-N \sum_{i} \alpha_{i} \ln \alpha_{i}=\frac{-N \sum_{i} \alpha_{i} \log _{2} \alpha_{i}}{\log _{2} e} \approx-N \sum_{i} \alpha_{i} \log _{2} \alpha_{i}=-N H(\alpha)
$$

For one sample of a sequence, the corresponding estimation of number $\log _{2} Q$, as follows: part of a Block Number Code is as follows:

$$
k_{Q}=k_{\text {frame }} / N=-\sum_{i} \alpha_{i} \log _{2} \alpha_{i} \equiv H(\alpha)
$$

$$
-N \sum_{i=1}^{n} \alpha_{i} \log _{2} \alpha_{i}>\log _{2} \frac{N !}{N_{1} ! \ldots N_{n} !}
$$

The above-obtained relation is, by the upper As for the left-hand part of an inequality, we have

$$
-\sum_{i} N_{i} \log _{2} \frac{N_{i}}{N}=\sum_{i} \log _{2}\left(\frac{N}{N_{i}}\right)^{N_{i}}=\log _{2} \prod_{i} \frac{(N)^{N_{i}}}{N_{i}}=\log _{2} \frac{\prod(N)^{N_{i}}}{\prod N_{i}}=\log _{2} \frac{(N)^{N}}{\prod_{i} N_{i}}
$$

Analyzing the polynomial formula [22]

$$
\left(X_{1}+X_{2}+\cdots+X_{n}\right)^{N}=\sum_{K_{1}+K_{2}+\cdots+K_{n}=N} \frac{\left(N_{1}+N_{2}+\cdots+N_{n}\right) !}{N_{1} ! N_{2} ! \cdots N_{n} !} X_{1}^{N_{1}} X_{2}^{N_{2}} \cdots X_{n}^{N_{n}}
$$

(here, $K_{i}$ is manifold numbers sets, subordinated by the requirement $\prod_{i} K_{i}=N$, for $i=\overline{1, n}$ ), we observed that the different sets media are the natural numbers $K_{i}$. In our set, $K_{i}=N_{i}$ (that is possible to realize, as $\left.\sum_{i} N_{i}=N\right)$. In addition, this combination gives only one possible addend of this sum. Therefore, it is obvious that the sum (3) will be larger than its non-negative composite, namely:

$$
\left(X_{1}+X_{2}+\cdots+X_{n}\right)^{N}>\frac{\left(N_{1}+N_{2}+\cdots+N_{n}\right) !}{N_{1} ! N_{2} ! \cdots N_{n} !} X_{1}^{N_{1}} X_{2}^{N_{2}} \cdots X_{n}^{N_{n}}
$$

If the equation $X_{i}=N_{i}$ is also accepted, then we shall have the following:

$$
\left(N_{1}+N_{2}+\cdots+N_{n}\right)^{N}>\frac{\left(N_{1}+N_{2}+\cdots+N_{n}\right) !}{N_{1} ! N_{2} ! \cdots N_{n} !} N_{1}^{N_{1}} N_{2}^{N_{2}} \cdots N_{n}^{N_{n}}
$$

$$
\text { or } \quad \frac{N^{N}}{\prod_{i} N_{i}^{N_{i}}}>\frac{N !}{\prod_{i} N_{i} !} \text {. }
$$

Having compared equations (3) and (8), at the logarithm basis more than one, it is rightly to note that

$$
\log _{2} \frac{N^{N}}{N_{1}^{N_{1}} \ldots N_{n}^{N_{n}}}>\log _{2} \frac{N !}{N_{1} ! \ldots N_{n} !},
$$

as it was necessary to prove.

\section{STATISTICS OF SYSTEM SOURCES ACTIVITIES IS A PRIORI UNKNOWN}

In this case, the sequence set and its positions, i.e., distribution between separate source samples, are not fixed. Therefore, besides an arrangement code by a size $k_{Q}$, it is also necessary to present the information on its length by a size $k_{q}$ and the sequence sources set by a size $k_{r}$, since the activity distribution $\left\{\alpha_{i}\right\}$ a priori are not known. That is, the sequence servicing information 


$$
k_{\text {frame }}=k_{Q}+k_{q}+k_{H} .
$$

However, in practice, for the frame synchronization facilitation, it is necessary to work with sequences of a stationary value of length, in particular, from stationary values of both measurement data and servicing parts. Contrary to the above parsed structure, it is thus impossible, shaping a servicing part, to do without the Set and the Block Number Codes, whose digit capacities $k_{\mu}$ and $k_{Q}$, accordingly, are fixed.

Let us assume that the sequence shaping ceases at the sequence information part or the Block Number Code digit grid filling.

1) Let the first sequence of Data Set be filled. The Set Code length $k_{u}$ is determined by an information part length value of $N$, which should be large enough, nevertheless, from physical requirements, restricted. Its value should be such that the least fissile of the totality sources has filled even in one position of sequence of Data Set part on the one hand and limits by a value practically implemented digit grids of a Block Number Code $k_{Q}$ - on the other hand. Thus,

$$
1 / \alpha_{i \min } \leq N_{\max } \leq k_{Q} / H(\alpha) .
$$

The probability that the Set Code is filling faster than the Block Number Code, coincides with the probability that a length of the sequence of Data Set part exceeds the selected one. It is neglectfully small, because the Block Number Code length $k_{Q}$ optimality is close to entropy. Thus:

$$
\operatorname{Pr}\left\{N>N_{\max }\right\}=\operatorname{Pr}\left\{\left[H(\alpha)-\frac{k_{a}}{N}\right]>\Delta\right\}<\varepsilon,
$$

where $\varepsilon$ and $\Delta$ are arbitrary, certainly given positive small values.

$$
\underset{k_{Q} \rightarrow \infty}{\lim k_{H}}=\lim _{k_{Q} \rightarrow \infty} \frac{\log _{2} n}{k_{Q}}\left\{\frac{k_{Q}}{H(\alpha)} \log _{2}\left[1+\frac{n H(\alpha)}{k_{Q}}\right]+n \log _{2}\left[1+\frac{k_{Q}}{n H(\alpha)}\right]\right\}=0 .
$$

Thus, this enumeration method is asymptotically effective at a sequence considerable size of $N$, because the minimum possible expenditures on an enumeration cannot be less than the entropy value $[24,25]$. Such an approach may be used at designing of intelligent devices [26-28].

\section{ACTIVITY ESTIMATION BASEMENT}

Let us suppose that the data compression of the measurement system is based on the adaptive
The Block Number Code binary digits number, which is necessary on one selective sample value, is determined by totality sources activities entropy. Thus, it will be a maximum at the equiprobable activities distribution. Hence, at a given length of a Block Number Code, it is possible to get the sequence of Data Set part selective values amount, at which there will already be the filling

$$
N_{\min }=\frac{k_{Q}}{\log _{2} n} .
$$

2) The first Set Code digit grid is filled. Thus, if positions of the sequence of Data Set part are not yet exhausted, then the remainder of positions can be allocated on the "shadow" interrogation or other additional information.

At a Set Code shaping, similarly to equation (2), here it is necessary to enter one more numeral boundary [23], if only to designate the unused sequence of Data Set part positions, that is

$$
k_{H}=\log _{2} \frac{(N+n) !}{N ! n !} \approx N \log _{2} \frac{N+n}{N}+n \log _{2} \frac{N+n}{n}
$$

The limiting Set Code length is determined by the greatest possible sequence information part selective samples values $N_{\max }$

$$
k_{H \text { max }}=N_{\text {max }} \log _{2} \frac{N_{\text {max }}+n}{N_{\text {max }}}+n \log _{2} \frac{N_{\text {max }}+n}{n} .
$$

Expenditures, that are present on one selective sampling value, are as follows:

$$
k_{H}=\frac{k_{H \max }}{N}<\frac{k_{H \max }}{N_{\min }} .
$$

Taking into account the equations (10) and (11), we shall show an asymptotic optimality of the given approach, i.e.,

commutator principle. Then, all analog sources are sampled at a constant rate with the period $T$. In each sampling point, an adaptive commutator chooses among the total sources the most active one, i.e., the chosen source has the largest among other sources instant difference value, normalized with respect to its source analog measurement signal mean-square deviation (Fig.1). The samples of the rest sources are supposed to be redundant. The $i$-th most active source sampling value takes place at the $i$-th 
memory cell for the next sample time comparison, and the source activity manifestation is indicated at the $i$-th counter. Practically, the $i$-th source difference is estimated between the current sampling moment value and the previous activity manifestation value, which is picked from its memory cell. It is known [29] that the $i$-th source intensity

$$
\lambda_{i}=\sqrt{\frac{2}{\pi}} \frac{\omega_{1 i} \sigma_{1 i}}{X_{m}}
$$

here, $\omega_{i}$ and $\sigma_{i}$ are the $i$-th source mean-square frequency and mean-square deviation, respectively; $X_{m}$ is the sources totality maximum discretization error mean value.
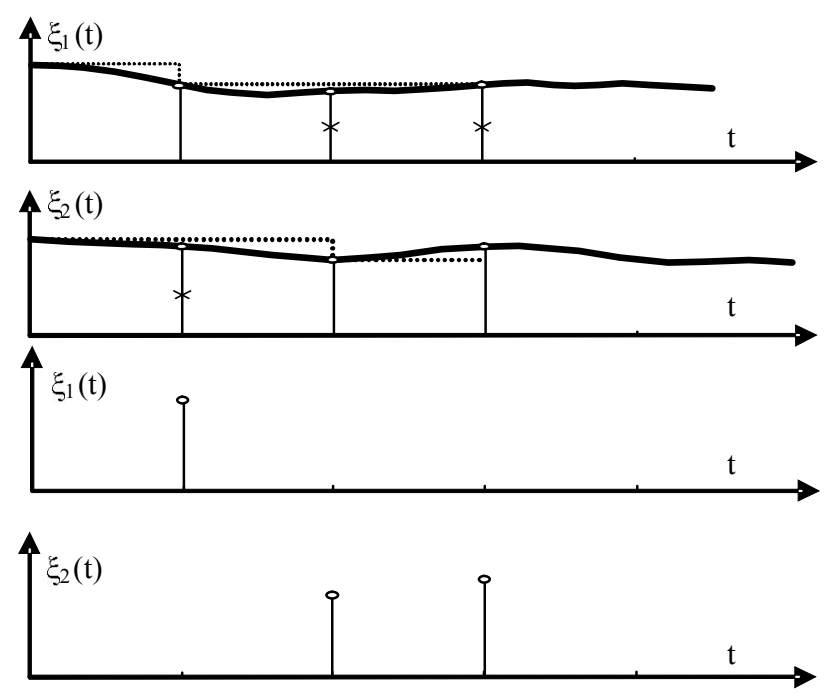

Fig. 1 - Sources activities estimation procedure.

In this case activities sequence is following: $1,2,2, \ldots$.

The $i$-th source relative activity $\alpha_{i}$ is determined as relative intensity

$$
\alpha_{i}=\lambda_{i} / \sum_{i} \lambda_{i}=\omega_{1 i} / \sum_{i} \omega_{1 i}=\omega_{1 i} / \omega_{\Sigma}
$$

here, $\omega_{\Sigma}$ is the system totality sources mean-square frequency (the last intensity to mean-square frequency transformation is possible at the equality of all sources discretization error values).

During the analysis time $T_{a}$, the current totality system sources absolute activity distribution $\left\{N_{i}\right\}, i=\overline{1, n}$ is formed at the $n$ counters. Therefore, it is possible to gather the non-redundant samples frame and its real time group code mapping. Moreover, due to all the sources counters contents, using the same algorithm, general object state mapping is realized. It was proved that the certain sources samples number corresponds to the unique single-valued coding combination (code value) [18]. The all sources activity distribution code (Block Number Code) is sent by the transmission link to the higher level of hierarchy.

\section{PROCESSING ALGORITHM DESCRIPTION}

The number of permutations is equal to

$$
M=\frac{N !}{N_{1} ! \ldots N_{n} !},
$$

here, $N_{i}$ is the $i$-th type symbols number among the $N$ sequence positions $(i=\overline{1, n}), \sum_{i} N_{i}=N$.

The permutation numerical coding algorithm is found on the chain division after the permutation place symbol [28]. It was noticed that a power of each subset is proportional to the ratio of a number of certain type of symbols to the total sequence positions number. Thus, for subset $S_{m}$ with $m$-type symbol at the first position

$$
M(m)=\frac{(N-1) ! N_{m}}{\prod_{i} N_{i} !}=M \frac{N_{m}}{N} .
$$

At the second step, each subset divides after the type symbol which takes the second position in the permutation. A power of a newly formed subset is proportional to the ratio of a certain type symbol number, which we meet from the second to the last position of the permutation to the total sequence positions number at this step, here it means to $(N-1)$. However, if at the second position we have the same $m$-type symbol, then the ratio is $\frac{N_{m}-1}{N-1}$, because at the second step we have yet only $\left(N_{m}-1\right) m$-type symbols and $(N-1)$ total positions, and so on. At a certain $j$-th step, some type of symbol cannot appear if its number has already exhausted. Such a procedure makes it possible to have a definite correspondence between a certain permutation set $S$ and its number of the natural row $0-(M-1)$. It was suspected, that the true enumerative coding would be if the sequence number is formed as follows:

$$
K(p)=\sum_{j=1}^{N} \sum_{i=1}^{x(j-1)} M_{j}(i)
$$

here, $M_{j}(i)$ is the $S_{j}(i)$ subset power value ; it should be noted that both the subset $S_{j}(i)$ permutations, and 
the analysed permutation $p$ have $(j-1)$ identical positions and the $i$-type symbol at the $j$-th position of permutation.

Therefore, the first $j$ permutation positions of a subset $S_{j}(i)$ are fixed. The number of such permutations defines probable permutations of the rest $(N-j)$ symbols. Within there is $\left[N_{m}-R_{m}(j)\right]-m$ type symbols $(m=\overline{1, n}, m \neq i)$ and $\left[N_{i}-R_{i}(j)-1\right]$ - $i$-type symbols; here, $R_{m}(j)$ is the number of $m$ - type symbols among the first $(j-1)$ positions of the permutation $p$.

Thus, the power of subset $S_{j}(i)(m \neq i)$

$$
M_{j}(i)=\frac{(N-j) !}{\prod_{m=1}^{n} N_{m} !} \prod_{r=1}^{j-1}\left[N_{X(r)}-R_{X(r)}(r)\right]\left[N_{i}-R_{i}(j)\right] .
$$

The kernel of the last expression (14)

$$
D_{j}=\frac{(N-j) !}{\prod_{m=1}^{n} N_{m} !} \prod_{r=1}^{j-1}\left[N_{X(r)}-R_{X(r)}(r)\right] .
$$

We can note that

$$
\left\{\begin{array}{l}
D_{1}=\frac{(N-1) !}{\prod_{m=1}^{n} N_{m} !} \\
D_{j+1}=\frac{D_{j}}{(N-j)}\left[N_{X(j)}-R_{X(j)}(j)\right]
\end{array}\right.
$$

Then,

$$
K(p)=\sum_{j=1}^{N} D_{j}\left[\sum_{i=a}^{b} N_{j}-C_{j}\right] \text { and } C_{j}=\sum_{i=a}^{b} R_{i}(j),
$$

here, $C_{j}$ is the quantity of symbols whose numbertype is less than the symbol number-type located at the $j$-th position of the permutation.

This algorithm can be used if the absolute activities values $\left\{N_{i}\right\}$ are known. Therefore, this is convenient for the activities distribution reflection. This is the so-called Set Code.

Non-redundant samples mapping of all the sources during the real appearance of the measurement data corresponds with the unknown activities values $\left\{N_{i}\right\}$ at the coding word formation $[18,27.28]$. This is the so-called Block Number Code.

For this case, it was noticed that each $j$-th position counted from the beginning might be considered as the $l$-th position from the end. Thus,

$$
l=N-j+1 .
$$

The kernel (15) corresponding to the $j$-th position from the beginning is the same for the $l$-th position from the end

$$
D_{l}=D_{j}
$$

Number $N_{i}(l)$ of $i$-type symbols among the last $l$ positions of a permutation

$$
N_{i}(1)=N_{i}-R_{i}(j) .
$$

Thus, using expressions (18)-(20) in equations (16) -(17), we receive a new algorithm [18]

$$
\begin{gathered}
K(p)=\sum_{l=1}^{N} D_{l} \sum_{i=a}^{b} N_{i}(l)=\sum_{l=1}^{N} K_{l}, \\
K_{l}=D_{l} \sum_{i=a}^{b} N_{i}(l), \\
\left\{\begin{array}{l}
D_{l}=\frac{D_{l-1}(l-1)}{N_{X(l)}(l)} \\
D_{1}=1 .
\end{array}\right.
\end{gathered}
$$

Permutation elements can be analysed in the order of their appearance and the number $N_{i}(l)$ is considered as the number of $i$-type symbols among the $l$ analysed. The permutation numbers obtained after (17) and (22) equations should be the same for the same input conditions, but with the opposite order of the appearance of elements.

Algorithm description is as follows [30]:

\section{Input:}

$\mathrm{N}$ - quantity of sequence positions

$\mathrm{Ni}[]$ - array of type symbols number among the $N$ sequence positions size of quantity of different values in sequence

X[] - set of values to encode size of $N$

\section{Output:}

$\mathrm{Kp}$ - value of kernel $\mathrm{K}(\mathrm{p})$

$\mathrm{R}[]$ - array of numbers of $i$ - type symbols among the permutation $p$

\section{Algorithm's initialization:}

$K p \leftarrow 0$

$D \leftarrow 1$

$\mathrm{R}[] \leftarrow\{0\}$

\section{$1: \mathrm{R}[\mathrm{X}[1]] \leftarrow \mathrm{R}[\mathrm{X}[1]]+1$}

Kernel $p$ value calculation when $l=1$

2: for $i=1$ to $X[1]-1$

3: \{

4: $\quad K p \leftarrow K p+N i[i]-R[i]$

5:\} 
6: if $N>1$ then

7: \{

Comment: Kernel $\quad p$ value calculation when $l>1$

8: $\quad$ for $l=2$ to $N$

9:

10: $\quad \mathrm{R}[\mathrm{X}[l]] \leftarrow \mathrm{R}[\mathrm{X}[l]]+1$

Comment: $D$ assign value of $D_{l}=\frac{D_{l-1}(l-1)}{N_{X(l)}(l)}$

Comment: Acc assign value of $\sum_{i=a}^{b} N_{i}(l)$

12:

13:

14:

15:

$16:$

17:

18: $\quad\}$

19: $\}$

Such servicing information perfectly corresponds to the information entropy of the object sources and may be used for an express analysis of the current state of the object. At each step of analysing the information state of the investigated object, the most active sensor is chosen. An output analog signal of any sensor is conditioned and a certain address number is prescribed to it. To illustrate the process of analysing let us consider a simplified example of sensor network which consists of three sources, and the analyzing period consist of six steps (i.e., $n=3$, $N=6$ ). Let us suppose that we received the activities sequence as follows: $2,1,1,3,1,3$. This means that the first active sensor has number 2 and the last has number 3. After formula (22), in this case we obtain the following:

$$
\begin{aligned}
& D_{1}=1, \\
& D_{2}=D_{1} \cdot 1 / N_{X(2)}(2)=1 \cdot 1 / 1=1, \\
& D_{3}=D_{2} \cdot 2 / N_{X(3)}(3)=1 \cdot 2 / 2=1, \\
& D_{4}=D_{3} \cdot 3 / N_{X(4)}(4)=1 \cdot 3 / 1=3, \\
& D_{5}=D_{4} \cdot 4 / N_{X(5)}(5)=3 \cdot 4 / 3=4, \\
& D_{6}=D_{5} \cdot 5 / N_{X(6)}(6)=4 \cdot 5 / 2=10 .
\end{aligned}
$$

The code (i.e., group number) that corresponds with the situation is following (21):

$$
\begin{aligned}
& K(p)=\sum_{l=1}^{N} D_{l} \sum_{i=1}^{X(l)-1} N_{i}(l)=1 \cdot 0+1 \cdot 0+1 \cdot 0+3(2+1)+ \\
& +4 \cdot 0+10(3+1)=40 .
\end{aligned}
$$

It was formed in real time. Another order of addresses results in a different group number. The information entropy estimation (4) is calculated as follows:

$H(\alpha)=-\frac{1}{2} \log _{2} \frac{1}{2}-\frac{1}{6} \log _{2} \frac{1}{6}-\frac{1}{3} \log _{2} \frac{1}{3}=1,46$ bit,

here, $N_{1}=3, N_{2}=1, N_{3}=2, N=\sum_{i} N_{i}=6$, then $\alpha_{i}=N_{i} / N$ and $\alpha_{1}=1 / 2, \alpha_{2}=1 / 6, \alpha_{3}=1 / 3$. Therefore, a set of group numbers describes an object information state entropy.

\section{SYSTEM OPERATION DESCRIPTION}

The output signal (Fig. 2) of each analog sensor (Source) is passed to the proper $\left(l_{1} \ldots l_{n}\right)$ input of the Activity Analyzing Unit (AAU).

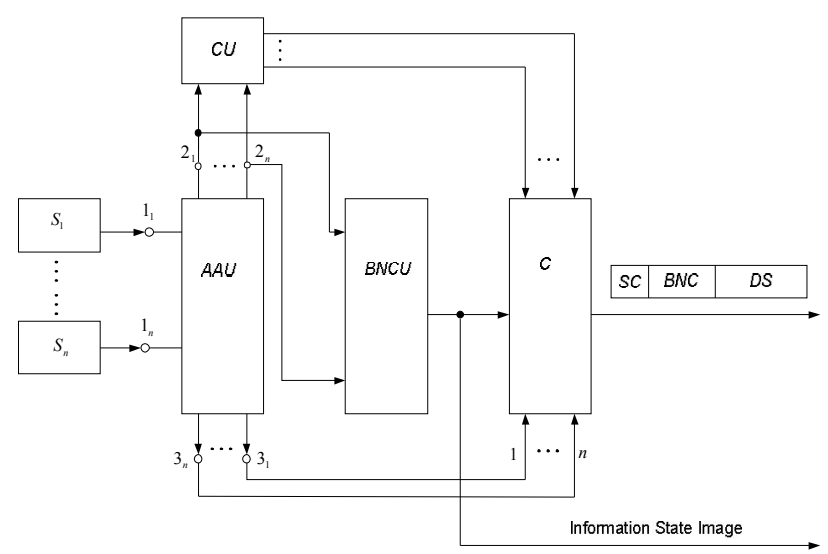

a)

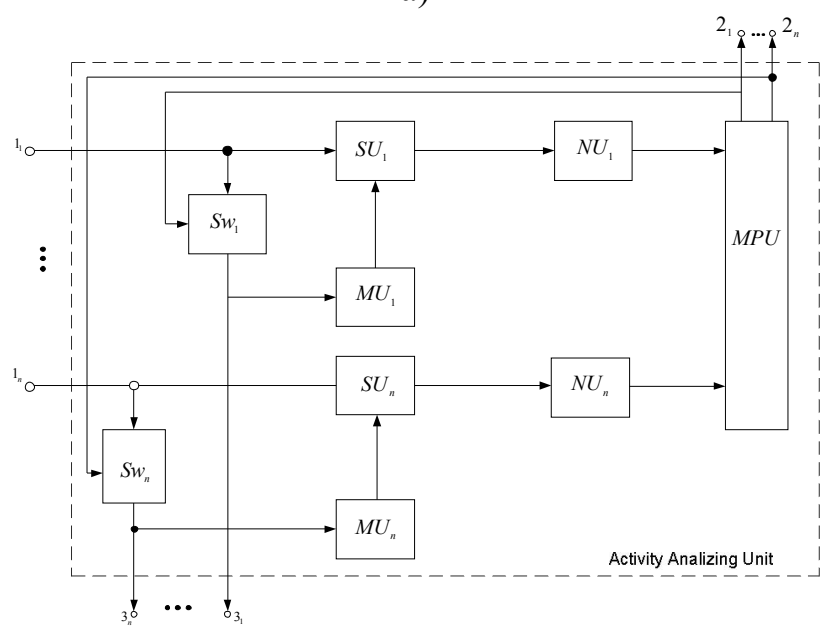

b)

Fig. 2 - Simplified scheme of system (a) and activities analyzing unit operation (b).

At the very beginning, the first samples of all sensors are put through the open Switch (Sw) to its corresponding Memory Unit (MU). At the next (i.e., second) sampling step, all the switches are closed. The sample of each sensor is passed to the 
Subtraction Unit (SU) as a diminishing value, while its previous sample is a subtrahend value. A deviation is normalized by division operation in Normalization Unit (NU) and follows to the Maximum Polling Unit (MPU). In this unit, the source is chosen with the maximum normalized deviation, which is nominated as the most active among the totality. MPU makes it possible to open the switch $\mathrm{Sw}$ in this active source channel $\left(2_{1} \ldots 2_{n}\right)$ and its sample removes the previous one in its corresponding Memory Unit (MU) as well as it is passed through proper outputs $\left(3_{1} \ldots 3_{n}\right)$ to the Coder (C). At the next (i.e., third) sampling step described above, operations are repeated. Note, that only for an active source channel the subtrahend was changed.

Then, during $\mathrm{N}$ sampling steps, both the amount of the manifested source activities is accumulated in the Counting Unit $(\mathrm{CU})$ and BNC Unit (BNCU) forms the Block Number Code in real time.

Following the arrival of the $\mathrm{N}$-th last nonredundant sample of the Data Set from the totality active sources the output frame is supplemented by prepared Block Number Code and by the proper Set Code. This frame is formed by Coder and is passed to the system modem for transmission. Separately, the $\mathrm{BNC}$ is also transmitted at a higher level of control hierarchy for the next information state examination

\section{CONCLUSIONS}

The binary digit rate demand at the sequence length number $N \rightarrow \infty$ is guided to the group source entropy productivity. The Block Number Code is a uniform unique number depending on the activities of the system sources. At the receiver side, the decoded number is used for the samples distribution from the memory register on the relevant channels. Thus, the sequence number also describes the totality sources activities state, i.e., the servicing object state. This estimation may be used for the next representation at a higher hierarchy level. This algorithm does not need to know the sources statistics before encoding and works in a real time. The Set Code is formed simultaneously with the Block Number Code formation.

\section{REFERENCES}

[1] J. H. Holland, Adaptation in Natural and Artificial System, MIT Press, Cambrige, Massachusetts, 1992, $257 \mathrm{p}$.

[2] A. R. Alameldeen, D. A. Wood, Adaptive cache compression for high-performance processors, in Proceedings of the 31st Annual International Symposium on Computer Architecture, Munchen, Germany, (19-23 June 2004), pp. 212-223.
[3] R. Curry, W. V. Velde, J. Potter, Nonlinear estimation with quantized measurements PCM, Predictive Quantization, and Data Compression, IEEE Transactions on Information Theory, (16) 2 (1970), pp. 152161.

[4] J. Wan, H. Yan, H. Suo, F. Li, Advances in Cyber-physical systems research, KSII Transactions on Internet and Information Systems, (5) 11 (2011), pp. 1891-1908.

[5] D. Slepian, Permutation modulation, Proceedings of IEEE, (53) 3 (1965), pp. 228236.

[6] C. C. Y. Poon, Y.-T. Zhang, S.-D. Bao, A novel biometrics method to secure wireless body area sensor networks for telemedicine and m-health, IEEE Communications Magazine, (44) 4 (2006), pp.73-81.

[7] C. Bandt, B. Pompe, Permutation entropy: a natural complexity measure for time series, Phys. Rev. Lett., (88) 17 (2002), pp. 174102.

[8] J. M. Amigó, M. B. Kennel, L. Kocarev, The permutation entropy rate equals the metric entropy rate, Physica D: Nonlinear Phenomena, (210) 1-2, (2005), pp. 77-95.

[9] New Directions in Wireless Communications Research, edited by Vahid Tarokh, Springer Verlag US, 2009, $465 \mathrm{p}$.

[10] T. Berger, Minimum entropy quantizers and permutation codes, IEEE Transactions on Information Theory, (28) 2 (1982), pp. 149157.

[11] J. Amigo, Permutation Complexity in Dynamical Systems, Springer Series in Synergetic, Springer Verlag, Berlin /Heidelberg /Dordrecht /London /New York, 2010, 348 p.

[12] A. El Saddik, M. Orozco, Y. Asfaw, S. Shirmohammadi, A novel biometric system for identification and verification of haptic users, IEEE Transactions on Instrumentation and Measurement, (56) 3 (2007), pp. 895-906.

[13] T. E. Avery, H. E. Burkhart, Forest Measurements, Waveland Press, Inc., Long Grove, Illinois, USA, 2015, 455 p.

[14] P. Abina, K. Dhivyakata, L. Suganya, S. M. Praveena, Biometric autentification system for body area network, International Journal of Advanced Research in Electrical, Electronics and Instrumentation Engineering, (3) 3 (2014), pp. 7954-7963.

[15] N. Houmani, S. Garcia-Salicetti, B. Dorizzi, A novel personal entropy measure confronted with online signature verification systems' performance, in Proceedings of the 2nd IEEE International Conference on Biometrics: Theory, Applications and Systems, Arlington, VA, USA, (Sept. 29 - Oct. 1, 2008), pp. 1-6. 
[16] C. H. Bennett, F. Bessette, G. Brassard, L. Salvail, J. Smolin, Experimental Quantum Cryptography, Journal of Cryptology, (5) 1 (1992), pp. 3-28.

[17] Y. Cao, W.-w. Tung, J. B. Gao, V. A. Protopopescu, and L. M. Hively, Detecting dynamical changes in time series using the permutation entropy, Phys. Rev. E 70, (70) 4 (2004), pp. 046217.

[18] O. Ivakhiv, Measurement source enumerative coding, Control and Measuring Equipment, (50) (1993), pp. 12-22. (in Ukrainian).

[19] T. Dominyuk, O. Ivakhiv, P. Mushenyk, A. Kowalczyk, B. Stadnyk, Serving object information state estimation, in Proceedings of the IMEKO-TC7 Symposium on Measurement Science of the Information Era, Cracow, Poland, (June 25-27, 2002), pp. 131-134.

[20] T. Berger, F. Jelinek, J. Wolf, Permutation codes for source coding, IEEE Transactions on Information Theory, (18) 1 (1972), pp. 160169.

[21] J. K. Karlof, Permutation codes for the Gaussian channel, IEEE Transactions on Information Theory, (35) 4 (1989), pp. 726732.

[22] N. Vilenkin, Combinatorics, Moscow, Nauka Press, 1969, 197 p. (in Russian).

[23] E. C. Posner, I. Eisenberger, T. O. Anderson and W. A. Lushbaugh, Data Compressor, US Patent, \# 31369.222, 340-172.5.

[24] T. Cover, J. Thomas, Elements of Information Theory, John Wiley \& Sons. Inc., New York/Chichester/Brisbane/Toronto/Singapore, 1991, $542 \mathrm{p}$.

[25] R. Behota, O. Ivakhiv, Z. Kushnir, Y. Tolopko, Enumerative source coding asymptotic optimality, Control and Measuring Equipment, (46) (1989), pp. 3-9. (in Russian).

[26] O. Ivakhiv, A. Kowalczyk, R. Velgan, Intelligent programmable measurement system, in Proceedings of the XVI IMEKO World Congress, Vienna, Wien, Austria (September 25-28, 2000), vol. IX, topic 30 - Artificial Intelligence in Measurement Techniques, pp. 341-345.
[27] T. Dominyuk, O. Ivakhiv, Ye. Pokhodylo, B. Stadnyk, R. Velgan, Quality estimation marking, 47. Internationales Wissenschaftliches Kolloquium Tagunsband. Maschinenbau und Nanotechnik - Hochtechnologien des 21. Jahrhunderts. Mechanical Engineering Nanotechnology, Ilmenau, Germany (2326.09.2002), pp. 419-420.

[28] O. Ivakhiv, P. Mushenyk, O. Bazylevych, Intelligent system for testing of object information state, in Proceedings of the 6-th IEEE International Conference on Intelligent Data Acquisition and Advanced Computing Systems: Technology and Applications IDAACS'2011, Prague, Czech Republic (September 15-17, 2011), 2011, vol. 2, pp. 633636.

[29] I. Kalashnikov, V. Stepanov, A. Churkin, Adaptive Data Gathering and Transmitting Systems, Moscow, Energiya, 1975, 240 p. (in Russian).

[30] O. Ivakhiv, P. Mushenyk, Y. Hirnyak, Intelligent analyzing system, Sensors \& Transducers Journal, (24) Special Issue (2013), pp. 43-49.

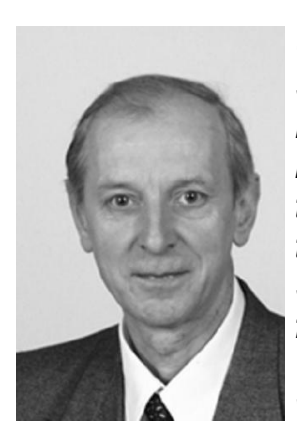

Orest Ivakhiv received his M.A. Sc. in Electrical Engineering from Lviv Technical University, Ukraine, in 1968, Ph.D. in Comunications from Moscow Aviation Institute, Russia, in 1979 and Dr. Sci. in Electrical Engineering from Lviv Polytechnic National University in 2002. Orest Ivakhiv was a visiting professor at the Department of Electrical and Computer Engineering at University of Toronto, Canada, in 1994. Since 1968 professor O. Ivakhiv is a faculty member at the Computer Technology, Automation and Metrology Institute of Lviv Polytechnic National University. He has 300 publications, including 11 patents, 2 monographs, 6 textbooks. His areas of scientific interests are electrical measurement and instrumentation, adaptive data processing and compression, enumerative coding and mechatronics. 